\title{
Compact, Broadband, and Omnidirectional Antenna Array for Millimeter-Wave Communication Systems
}

\author{
Yuanzhi Liu ${ }^{1}$, Mustapha C. E. Yagoub ${ }^{1}$ (D) \\ ${ }^{1}$ School of Electrical Engineering and Computer Science, University of Ottawa, Ottawa K1N 6N5, Canada, \\ yliu600@uottawa.ca,myagoub@uottawa.ca
}

\begin{abstract}
In this paper, a millimeter-wave printed quadrupole antenna is proposed. It consists of two symmetric printed dipoles that make the quadrupole radiating power omnidirectionally. Such an arrangement eliminates the influence of the ground as reflector. Simulation results show that the quadrupole antenna exhibits a broad impedance bandwidth and an enhanced gain compared to conventional dipoles. More interestingly, the configuration of the quadrupole includes an integrated feed network, making the design compact. Next, a novel omnidirectional array was designed, simulated, fabricated, and measured, exhibiting enhanced features like compactness, broad bandwidth, high gain, high efficiency, low cross-polarization, low cross-polarization level, low cost, and low profile.
\end{abstract}

Index Terms - millimeter-wave; quadrupole; dipole; omnidirectional; broadband; antenna array

\section{INTRODUCTION}

The millimeter-wave (mm-wave) communication technique, which can use enormous underutilized bands beyond the traditional licensed bands to overcome the challenge of global bandwidth shortage, has been recognized as a key technology for the future communication systems [1], [2]. In 2015, several mm-wave bands including $24.25-27.5 \mathrm{GHz}, 37-40 \mathrm{GHz}$, and $66-76 \mathrm{GHz}$ for the fifthgeneration $(5 \mathrm{G})$ communications and beyond applications have been licensed by the International Telecommunication Union [3]. However, the path loss of mm-wave is much higher than that of lower frequencies [4], highlighting the design challenge of antenna arrays with high gain [5].

On the other hand, in places such as malls and stadiums, where users are randomly and uniformly distributed, omnidirectional antennas are desired [6]. However, there are only few reported omnidirectional antennas in these bands mainly because of the challenges a designer will face compared to designing directional antennas. For instance, the ground of the antenna needs to be properly placed or it can reflect radiated electromagnetic waves and degrade the expected omnidirectional pattern. A typical example is printed dipoles, in which the existence of a ground plane makes the dipoles inherently provide directional or quasi-omnidirectional power radiation [7]. As a result, this type of antenna is usually used as driven element to design Yagi-Uda antennas and arrays that can provide more focused beams [8]-[10]. Omnidirectional arrays are even more difficult to design because the extra feed networks also contain grounds. For these reasons, most of the designed 
omnidirectional antennas suffer from complex structure, bulky size, and low gain [11]-[13]. The 28 $\mathrm{GHz}$ omnidirectional antenna presented in [14] has a complex non-planar structure, a small gain of $2.08 \mathrm{dBic}$, and a narrow $-10 \mathrm{~dB}$ impedance bandwidth $(27-28.5 \mathrm{GHz}, 5.4 \%)$. The planar antenna presented in [15] achieved a better performance of bandwidth (57 - $64 \mathrm{GHz}, 11.6 \%)$, but its average gain is only $1.4 \mathrm{dBi}$.

In this paper, a novel printed quadrupole antenna configuration is proposed. It consists of two symmetric dipoles and a ground placed such that the antenna can radiate power omnidirectionally without being influenced by the ground. More interestingly, one can easily design an array based on the quadrupole antenna. Such a configuration allows the feed network being integrated inside the elements without increasing the overall size of the array. More importantly, no extra ground will be added, and omnidirectional radiation pattern will be maintained. Targeting the $5 \mathrm{G} 26 \mathrm{GHz} \mathrm{mm}$-wave band, the designed quadrupole antenna and its array were simulated by the Ansys-HFSS commercial software. The quadrupole exhibits a good impedance bandwidth of $2.7 \mathrm{GHz}$ and an enhanced gain of $5.6 \mathrm{dBi}$. Next, a $1 \times 6$ array fed by a microstrip line feed network was designed. With a very compact size of $10 \times 42.3 \mathrm{~mm} 2\left(0.87 \lambda_{0} \times 3.67 \lambda_{0}\right.$, with $\lambda_{0}$ the free space wavelength at $\left.26 \mathrm{GHz}\right)$, the array has a gain higher than $12 \mathrm{dBi}$ in the operating frequency range. Besides, a broad $-10 \mathrm{~dB}$ impedance bandwidth of $8.64 \mathrm{GHz}$ is achieved. Simulated results also show that both the single antenna and the array have high efficiency and low cross-polarization levels. The proposed antenna array was fabricated and measured; measurement results agree well with simulation ones. Despite their good performance, they also feature low profile, low cost, simple planar structure, and simple fabrication process.

\section{PRInTED QuAdrupole ANTENNA}

\section{A. Conventional Printed Dipole Antenna}

Fig. 1 shows a conventional half-wavelength dipole antenna operating at $26 \mathrm{GHz}$ printed on the Rogers RO 4003C laminate with a relative permittivity of 3.55 , a loss tangent of 0.0027 , and a substrate thickness of $0.203 \mathrm{~mm}$ (all the antennas presented in this paper are printed on this substrate). Half-wavelength dipoles, as we all know, are a type of omnidirectional antennas. However, the antenna shown in Fig. 1 has a directional radiation pattern as illustrated in Fig. 2 (In the specified coordinate system, the E- and H-plane are the $x z$ and $y z$ plane, respectively) because the ground works as a reflector as aforementioned. 


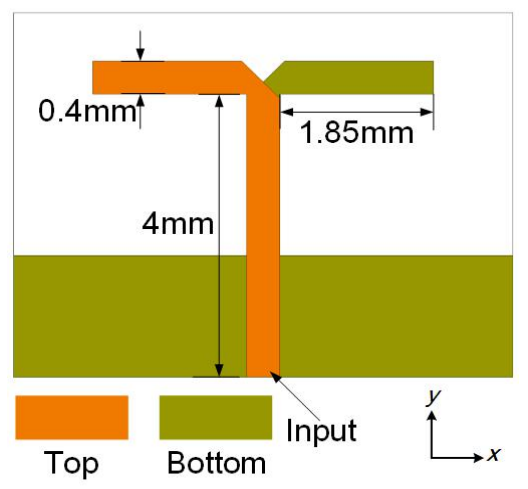

Fig. 1. Configuration of a conventional printed dipole antenna operating at $26 \mathrm{GHz}$.

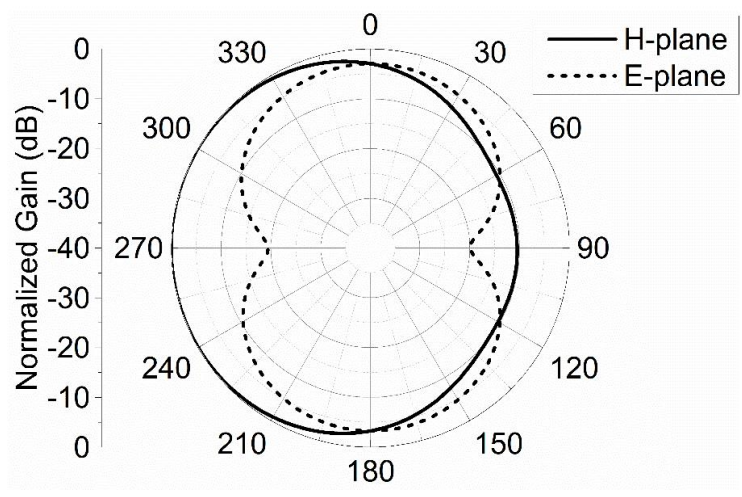

Fig. 2. Normalized gain of the conventional printed dipole antenna.

\section{B. Proposed Printed Quadrupole Antenna}

To achieve an omnidirectional radiation pattern, a second dipole was added symmetrically, such that the reflector is actually "inside" the antenna and the antenna can radiation power towards all the directions at H-plane. Previously the dipole can only radiate power toward one direction because of the existence of the ground. However, the ground is located inside the quadrupole such that it has no impact on the expected omnidirectional radiation pattern.

This configuration (Fig. 3) can be seen as a printed quadrupole antenna. Compared to existing designs, this antenna is of low profile, cost effective, and can be easily fabricated due to its simple fabrication process and planar structure. In fact, previous works used complicated structures such as substrate integrated waveguide (SIW) and multi-layer structures or multi-pole configurations implemented as radiation parts to design omnidirectional antennas [6][16][17]. Fed by a one-pointtwo microstrip line power splitter, the quadrupole antenna not only has an omnidirectional radiation pattern at its operating frequency, $26 \mathrm{GHz}$ in this design, but also exhibits a good -10dB impedance bandwidth of $2.7 \mathrm{GHz}$, an enhanced gain of $5.6 \mathrm{dBi}$, and a high radiation efficiency of over $95 \%$. All the above-simulated results are shown in Figs. 4 and 5. 


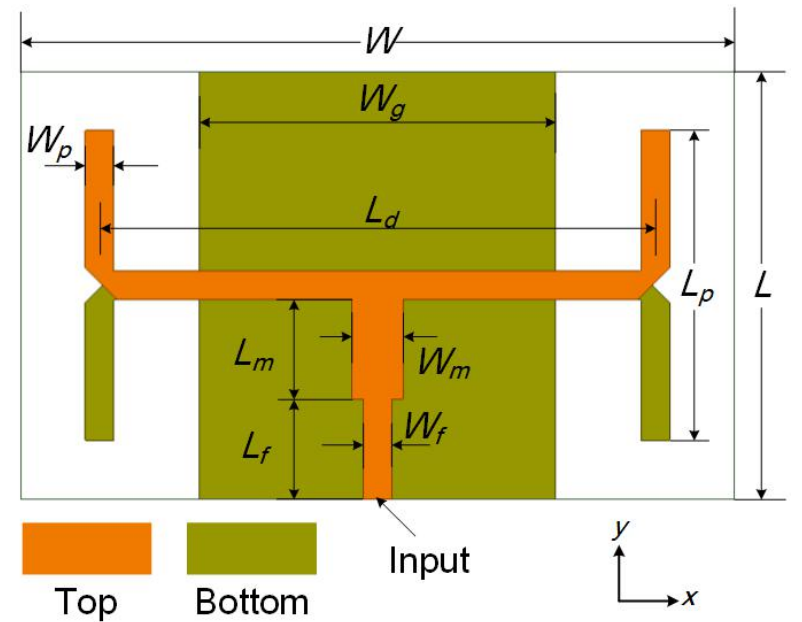

Fig. 3. Configuration of the proposed quadrupole antenna $\left(W_{f}=0.4 \mathrm{~mm}, L_{f}=1.7 \mathrm{~mm}, W_{m}=0.72 \mathrm{~mm}, L_{m}=1.1 \mathrm{~mm}, W_{p}=\right.$ $0.4 \mathrm{~mm}, L_{p}=4.34 \mathrm{~mm}, L_{d}=7.8 \mathrm{~mm}, W_{g}=5 \mathrm{~mm}, W=10 \mathrm{~mm}$, and $\left.L=6 \mathrm{~mm}\right)$.

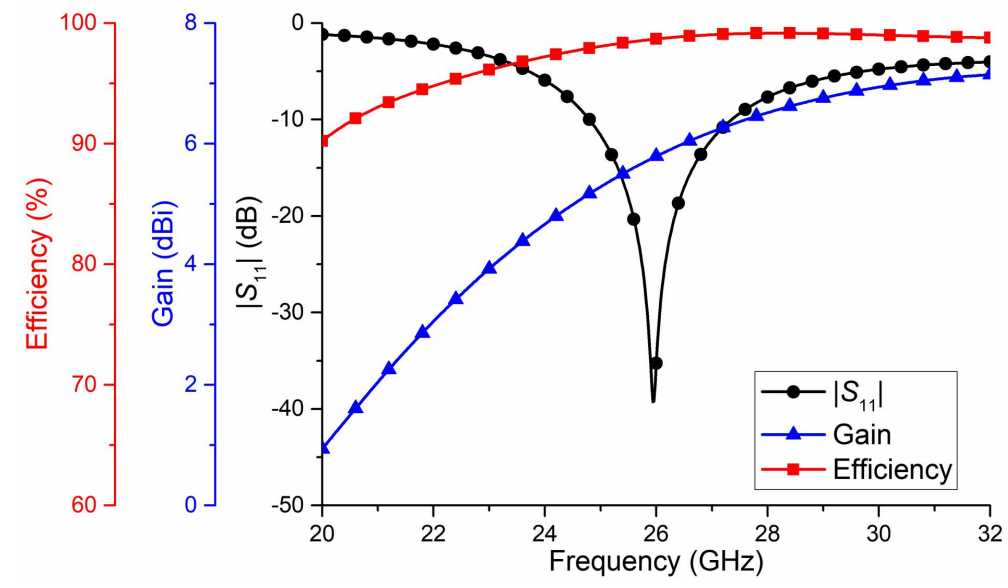

Fig. 4. $\left|S_{11}\right|$, gain, and efficiency of the proposed quadrupole antenna.

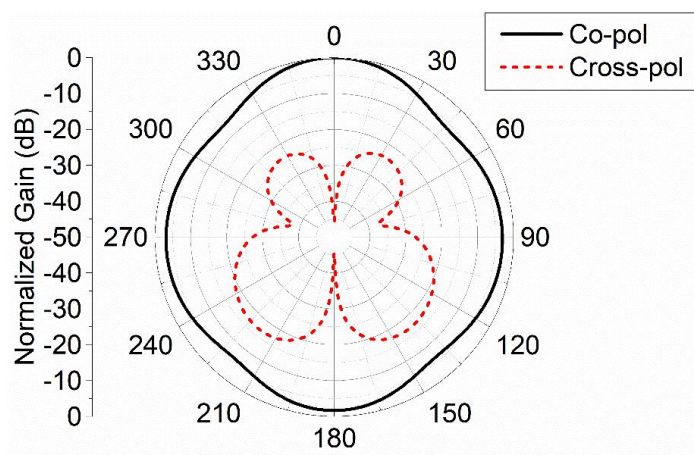

(a)

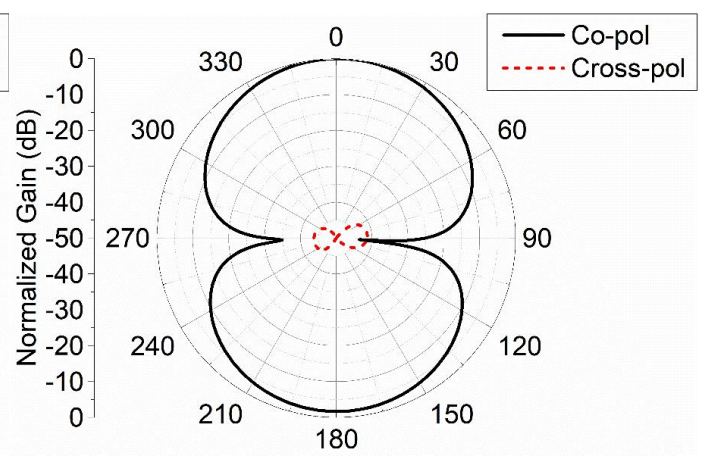

(b)

Fig. 5. Normalized gain of the proposed quadrupole antenna: (a). H- (xz) plane and (b). E- (yz) plane.

The distance between two symmetric dipoles is the main factor that influences the H-plane radiation pattern. Fig. 6(a) and Fig. 6(b) present the H-plane normalized gain and $\left|S_{11}\right|$ of the antenna with different $L_{d}$, respectively. According to the results, we can clearly see that the antenna is more omnidirectional with smaller $L_{d}$. Meanwhile, this parameter has an impact on the resonant frequency and impedance matching.

Brazilian Microwave and Optoelectronics Society-SBMO received 19 Nov 2020; for review 23 Nov 2020; accepted 16 March 2021 


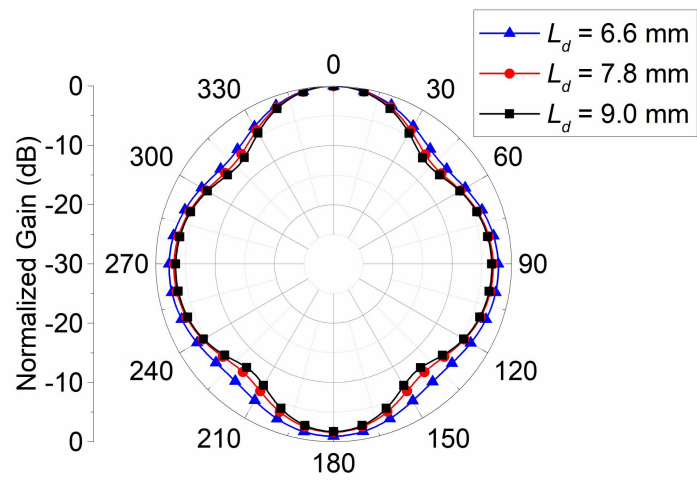

(a)

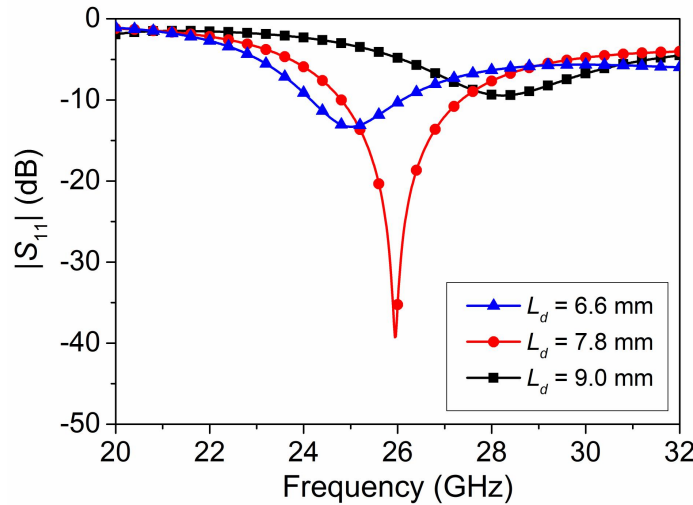

(b)

Fig. 6. The impact of $L_{d}$ on the performance of the proposed quadrupole antenna: (a). normalized gain and (b). $\left|S_{11}\right|$.

\section{Printed Quadrupole Antenna ArRay}

\section{A. Configuration of the proposed quadrupole antenna array}

To achieve an omnidirectional radiation pattern, an array of such a quadrupole antenna must be arranged along the current flow direction on dipoles. However, to feed it, we need to use a proper feed network. One might consider the conventional series-feed or corporate-feed networks, but neither will work as expected because there will be some issues. Despite the high losses and undesired power radiation of microstrip lines at such high frequencies [18], the feed network contains an extra ground, which will work as a reflector and thus, will degrade the expected omnidirectional radiation pattern of the array.

We, therefore, propose a feed network configuration that can be integrated inside the array. As shown in Fig. 7, a hybrid series- and corporate-feed network is designed and placed inside the quadrupoles. Such a feed network is tight so that it radiates less undesired power and has less loss compared to conventional ones. To achieve a uniform power distribution (of course unequal power distribution can also be adopted to supress sidelobes) and match the feed network with $50 \Omega$, the characteristic impedance of all the microstrip lines should strictly calculated. Each junction (Fig. 7) must be designed to split the incoming power by determined fraction.
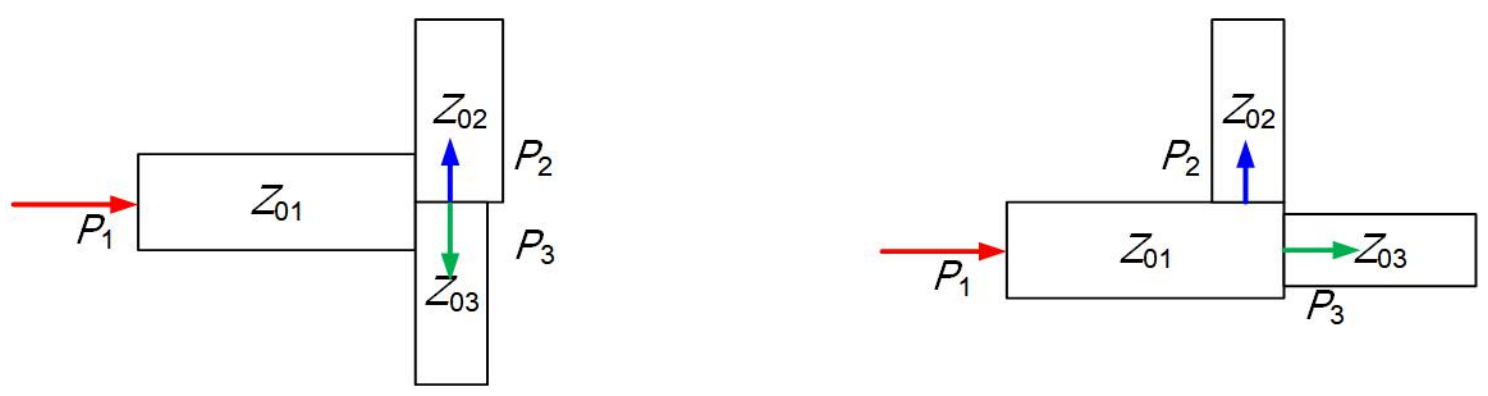

Fig. 7. Two types of T-junctions used in this work.

Power $P_{1}$ enters the T-junctions and is split into power $P_{2}$ and $P_{3}$. The ratio of the output powers will be related to the ratio of the characteristic impedance, namely $Z_{02}$ and $Z_{03}$, of the transmission 
lines:

$$
P_{2} / P_{3}=Z_{03} / Z_{02}
$$

Besides, by conservation of power, a lossless junction has

$$
P_{1}=P_{2}+P_{3}
$$

Furthermore, to ensure that the junction is matched at the input port requires that:

$$
Z_{01}=Z_{02} / / Z_{03}=\left(Z_{02} Z_{03}\right) /\left(Z_{02}+Z_{03}\right) \text {. }
$$

The first T-junction was designed to split power evenly. The ratio of divided power of the following T-junctions in order are 1:5, 1:4, 1:3, 1:2, and 1:1. In this case, all the quadrupole can receive power with equal amplitude. To avoid lines that have very large or small characteristic impedance, quarterwavelength transformers are implemented. The reason that they should be avoided is that microstrip lines with very large impedance bandwidth are wide, such that undesired power radiation would be significant. Microstrip lines with very small characteristic impedance, on the other side, could be too narrow to be fabricated. It is worth to emphasize that, intrinsically, higher gain can be achieved using more elements, but this will lead to using narrower microstrip lines due to the limitation of unequal microstrip power dividers. In this case, a six-element array is presented in this paper.

Adopting such a feed network, no extra parts are added, such that the array (Fig. 8) has a compact size, and the radiation pattern will certainly not be deformed. The optimized parameters of the transformers are given in TABLE I. The space of elements $\left(L_{d y}\right)$ is fixed to be one guided wavelength to avoid unnecessary bended lines since such space will not cause the appearance of grating lobes according to our simulated results. The top surface current distribution shown in Fig. 9 demonstrates that the elements are fed in phase. A prototype of the proposed antenna array was fabricated; Fig. 10 shows its top and back view. Note that the shape of the substrate was slightly adjusted to include the connector.

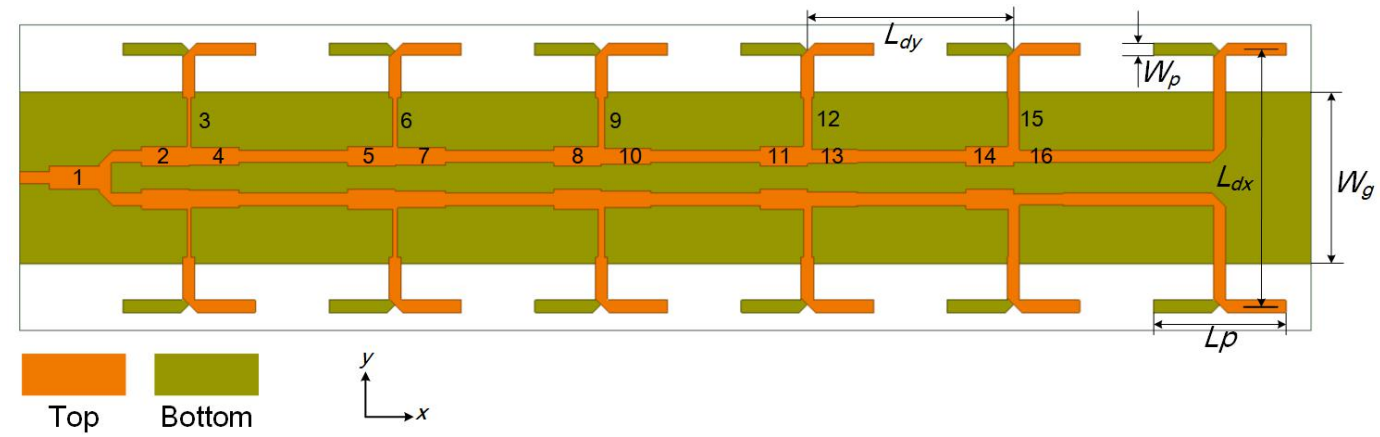

Fig. 8. Configuration of the proposed quadrupole antenna array $\left(W_{p}=0.4 \mathrm{~mm}, L_{p}=4.34 \mathrm{~mm}, L_{d x}=8.4 \mathrm{~mm}, L_{d y}=6.75 \mathrm{~mm}\right.$, $\left.W_{g}=5.6 \mathrm{~mm}\right)$. 


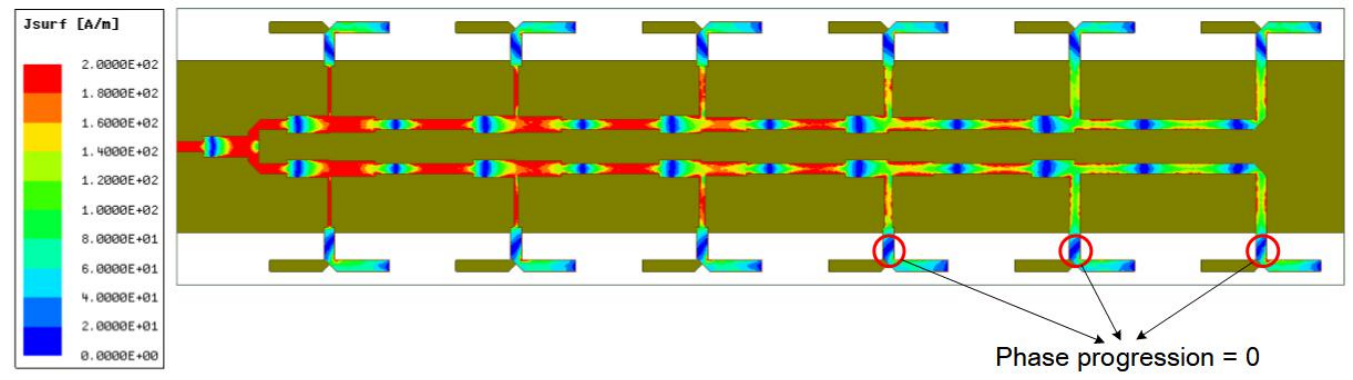

Fig. 9. Top surface current distribution of the proposed quadrupole antenna array.

TABLE I. IMPEDANCE TRANSFORMERS AND THEIR CORRESPONDING OPTIMIZED PARAMETERS

\begin{tabular}{cccc}
\hline Transformer & Characteristic Impedance $(\boldsymbol{\Omega})$ & Width $(\mathbf{m m})$ & Length $(\mathbf{m m})$ \\
\hline 1 & 35.4 & 0.738 & $\mathbf{1 . 6 3 0}$ \\
2 & 38.7 & $\mathbf{0 . 6 5 0}$ & $\mathbf{1 . 5 5 0}$ \\
3 & 94.9 & $\mathbf{0 . 1 3 0}$ & $\mathbf{1 . 7 7 5}$ \\
4 & 42.4 & $\mathbf{0 . 5 7 0}$ & $\mathbf{1 . 6 4 0}$ \\
5 & 38.7 & $\mathbf{0 . 6 5 0}$ & $\mathbf{1 . 5 5 0}$ \\
6 & 86.6 & 0.157 & $\mathbf{1 . 7 7 5}$ \\
7 & 43.3 & $\mathbf{0 . 5 5 2}$ & $\mathbf{1 . 6 5 0}$ \\
8 & 38.7 & $\mathbf{0 . 6 5 0}$ & $\mathbf{1 . 5 5 0}$ \\
9 & 77.5 & $\mathbf{0 . 2 0 0}$ & $\mathbf{1 . 7 7 5}$ \\
10 & 44.7 & $\mathbf{0 . 5 2 6}$ & $\mathbf{1 . 6 3 0}$ \\
11 & 38.7 & $\mathbf{0 . 6 5 0}$ & $\mathbf{1 . 5 5 0}$ \\
12 & $\mathbf{6 7 . 1}$ & $\mathbf{0 . 2 6 7}$ & $\mathbf{1 . 7 7 5}$ \\
$\mathbf{1 3}$ & $\mathbf{4 7 . 4}$ & $\mathbf{0 . 4 8 0}$ & $\mathbf{1 . 6 4 0}$ \\
$\mathbf{1 4}$ & $\mathbf{3 5 . 4}$ & $\mathbf{0 . 6 5 0}$ & $\mathbf{1 . 5 5 0}$ \\
$\mathbf{1 5}$ & $\mathbf{5 0}$ & $\mathbf{0 . 3 8 2}$ & $\mathbf{1 . 7 7 5}$ \\
$\mathbf{1 6}$ & $\mathbf{5 0}$ & $\mathbf{0 . 3 8 2}$ & $\mathbf{1 . 6 3 0}$ \\
\hline
\end{tabular}

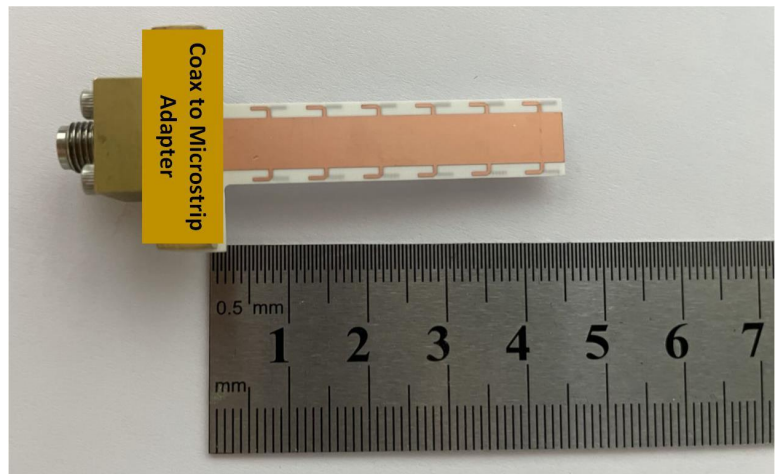

(a)

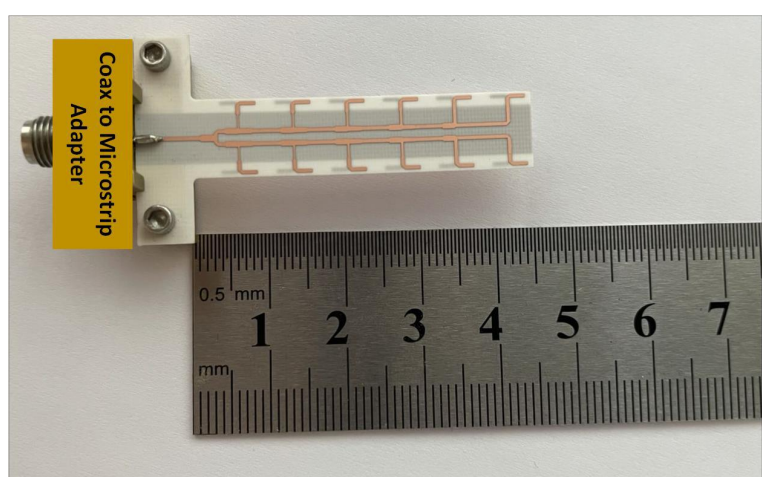

(b)

Fig. 10. A fabricated prototype of the proposed antenna array: (a). top view and (b). back view.

\section{B. Performance of the proposed quadrupole antenna array}

Figs. 11 and 12 present the simulated and measured results of the proposed quadrupole antenna array. For simulated results, the array exhibits a broad impedance bandwidth of $8.64 \mathrm{GHz}(21.68$ $30.33 \mathrm{GHz}$ ) for $|\mathrm{S} 11|<-10 \mathrm{~dB}$ and a high efficiency of over $90 \%$ in the frequency range of $21-32$ GHz. Besides, the array has a flat gain higher than $10 \mathrm{dBi}$ in the frequency range of $25.0-28.5 \mathrm{GHz}$ with a peak gain higher than $12 \mathrm{dBi}$ at $26 \mathrm{GHz}$. The gain out of that frequency range, however, drops 
significantly, mainly because of the deformation of the radiation pattern since the feed network can only provide equiphase power to the elements in a limited frequency range. The H-plane radiation pattern (Fig. 10(a)) of the array indicates its omnidirectional power radiation. Furthermore, the array has low cross-polarization levels especially at E-plane, whose simulated cross-polarization level is less than $-50 \mathrm{~dB}$. The maximum side lobe level at E-plane is $-12 \mathrm{~dB}$ since the array is uniformly fed.

The measured results agree well with the simulated results. The array has a measured $-10 \mathrm{~dB}$ impedance bandwidth of $9.38 \mathrm{GHz}(21.31 \mathrm{GHz}-30.69 \mathrm{GHz})$ and a measured gain of $12.08 \mathrm{dBi}$ at 26 GHz. The measured radiation patterns demonstrate the omnidirectional characteristics and low crosspolarization levels of the proposed array.

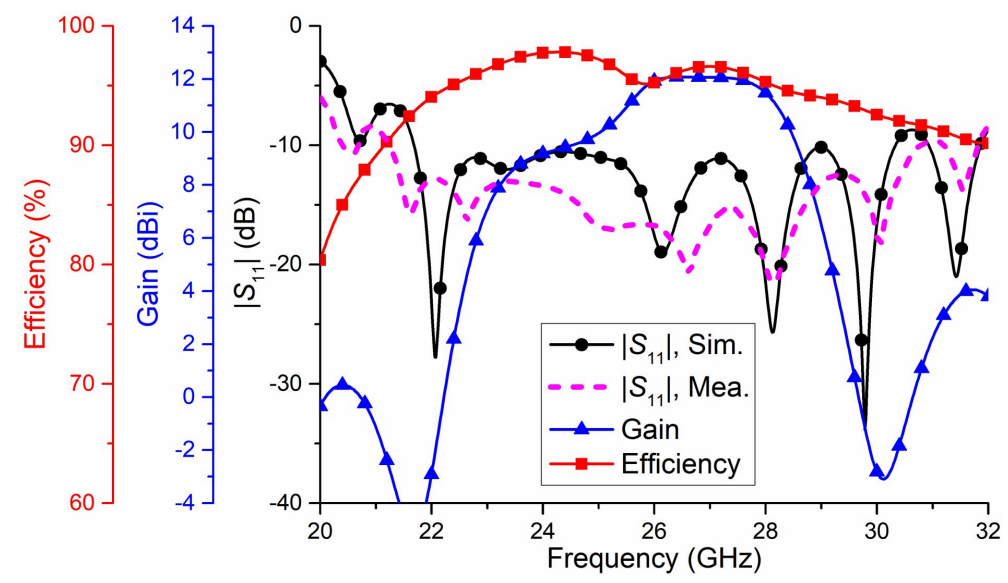

Fig. 11. $\left|S_{11}\right|$, gain, and efficiency of the proposed quadrupole antenna array.

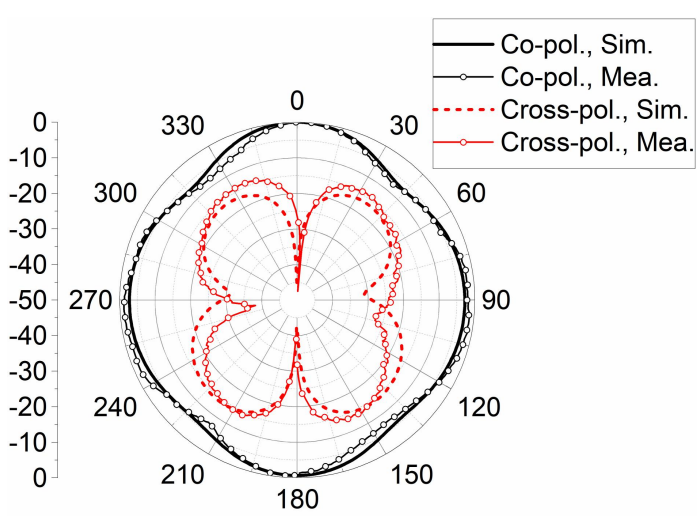

(a)

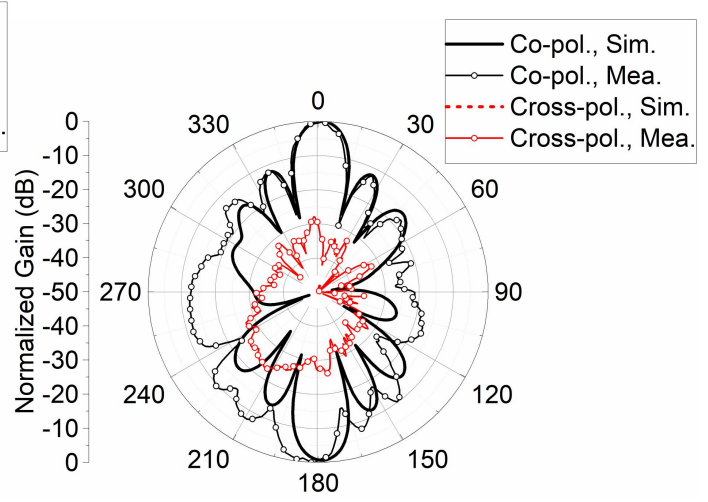

(b)

Fig. 12. Normalized gain of the proposed quadrupole antenna array: (a). H- (yz) plane and (b). E- (xz) plane. 
Journal of Microwaves, Optoelectronics and Electromagnetic Applications, Vol. 20, No. 2, June 2021 DOI: http://dx.doi.org/10.1590/2179-10742021v20i21114

C. Comparison with existing mm-wave omnidirectional antennas

To illustrate the good performance of the proposed array, Table II compares this work with some existing mm-wave omnidirectional antennas (including antenna arrays). Due to the challenge of designing mm-wave arrays as previously discussed, only few mm-wave omnidirectional arrays have been reported. According to that table, with a relative compact size and simple structure, this work achieved better performance in terms of impedance bandwidth and gain.

TABLE II. COMPARISON WITH EXISTING MM-WAVE OMNIDIRECTIONAL ANTENNAS

\begin{tabular}{|c|c|c|c|c|c|c|}
\hline Works & Operating Frequency $(\mathrm{GHz})$ & Gain & $\begin{array}{l}\text { Number of } \\
\text { Elements }\end{array}$ & Dimensions $\left(\mathrm{mm}^{2}\right)$ & Complexity & $\begin{array}{l}\text { Simulated/Measured } \\
\text { Results }\end{array}$ \\
\hline [6] & $24.00-29.00(18.8 \%)$ & $\approx 11.5 \mathrm{dBi}$ & 8 & $66 \times 15\left(5.72 \lambda_{0} \times 1.30 \lambda_{0}\right)$ & Moderate & Measured \\
\hline [14] & $27.00-28.50(5.4 \%)$ & $2.08 \mathrm{dBic}$ & 1 & $3.44 \times 3.44\left(0.32 \lambda_{0} \times 0.32 \lambda_{0}\right)$ & High & Simulated \\
\hline [16] & $25.80-29.30(12.7 \%)$ & $>12 \mathrm{dBi}$ & 4 & $68 \times 23.2\left(6.13 \lambda_{0} \times 2.09 \lambda_{0}\right)$ & Moderate & Simulated \\
\hline [19] & $27.00-29.00(7.1 \%)$ & $>10 \mathrm{dBi}$ & 8 & $100 \times 50\left(9.34 \lambda_{0} \times 4.67 \lambda_{0}\right)$ & Low & Simulated \\
\hline [20] & $57.00-64.00(11.6 \%)$ & $1.4 \mathrm{dBi}$ & 1 & $4.5 \times 4.3\left(0.91 \lambda_{0} \times 0.87 \lambda_{0}\right)$ & Low & Measured \\
\hline [21] & $33.70-38.50(13.2 \%)$ & $\approx 4 \mathrm{dBi}$ & 1 & $20 \times 5\left(2.41 \lambda_{0} \times 0.60 \lambda_{0}\right)$ & Low & Measured \\
\hline This work & $21.31-31.69(35.4 \%)$ & $12.08 \mathrm{dBi}$ & 6 & $42.3 \times 10\left(3.67 \lambda_{0} \times 0.87 \lambda_{0}\right)$ & Low & Measured \\
\hline
\end{tabular}

\section{CONCLUSION}

In this paper, a novel omnidirectional antenna configuration namely, a printed quadrupole, is proposed. Simulated results demonstrated its good performance in terms of gain, bandwidth, efficiency, and cross-polarization levels. More importantly, such a configuration can be easily used to design omnidirectional antenna arrays. A feed network has been integrated inside the antennas without increasing the overall size of the array and deforming the omnidirectional radiation pattern. The proposed array exhibits a broad impedance bandwidth, a high gain, a high efficiency, and low cross-polarization levels. The proposed antenna array was fabricated and measured; the measured results match well with the simulated results. The good performance was also demonstrated through successful comparison with published mm-wave omnidirectional antennas. In conclusion, the proposed array offers several other attractive features such as compactness, low cost, low profile, simple 2D geometry, and simple fabrication process.

\section{REFERENCES}

[1] T. S. Rappaport et al., "Millimeter wave mobile communications for 5G cellular: it will work!," IEEE Access, vol. 1, pp. 335-349, May, 2013.

[2] J. Zhang, X. Ge, Q. Li, M. Guizani, and Y. Zhang, "5G millimeter-wave antenna array: design and challenges," IEEE Wireless Commun., vol. 24, pp. 106-112, April 2017.

[3] M. J. Marcus, "5G and 'IMT for 2020 and beyond' [spectrum policy and regulatory issues]," IEEE Wireless Commun., vol. 22, pp. 2-3, Aug. 2015.

[4] W. L. Stutzman and G. A. Thiele, Antenna Theory and Design. 3rd Ed., J. Wiley \& Sons, 2012, pp. 109.

[5] Y. Liu, G, Bai, and M.C.E. Yagoub, "A 79GHz series fed microstrip patch antenna array with bandwidth enhancement and sidelobe suppression," Int. Conf. on Radar, Antenna, Microwave, Electronics and Telecomm., pp. 155-158, Tangerang, Indonesia, Nov. 2020

[6] C. X. Mao, M. Khalily, P. Xiao, T. W. C. Brown, and S. Gao, "Planar sub-millimeter-wave array antenna with enhanced gain and reduced sidelobes for 5G broadcast applications," IEEE Trans. Antennas Propag., vol. 67, pp. 160168, Oct. 2018

[7] N. O. Parchin, M. Shen, and G. F. Pedersen, "UWB MM-wave antenna array with quasi omnidirectional beams for 5G handheld devices," in IEEE Int. Conf. Ubiquitous Wireless Broadband, pp. 1-4, Nanjing, China, Oct. 2016.

[8] G. Bai, Y. Liu, and C. Liao, "A broad band high gain microstrip Yagi antenna array for mm-wave communication systems. Int. Conf. on Radar, Antenna, Microwave, Electronics and Telecomm., pp. 180-183, Tangerang, Indonesia, Nov. 2020. 
[9] F. Sun, F. Zhang, and C. Feng. "Wideband pattern reconfigurable printed-Yagi antenna array based on feed structure," $J$. of Microwaves, Optoelectronics Electromag. Applications, vol. 18, no. 2, 270-275. 2020.

[10] N. Kaneda, W. R. Deal, Yongxi Qian, R. Waterhouse and T. Itoh, "A broadband planar quasi-Yagi antenna," IEEE Trans. Antennas Propag., vol. 50, no. 8, pp. 1158-1160, Aug. 2002.

[11] Y. Fan, X. Liu, B. Liu, and R. Li, "A broadband dual-polarized omnidirectional antenna based on orthogonal dipoles," IEEE Antennas Wireless Propag. Lett., vol. 15, pp. 1257-1260, Nov. 2015.

[12] X. Dai, Z. Wang, C. Liang, X. Chen, and L. Wang, "Multiband and dual-polarized omnidirectional antenna for 2G/3G/LTE application," IEEE Antennas Wireless Propag. Lett., vol. 12, pp. 1492-1495, Nov. 2013.

[13] B. Li and Q. Xue, "Polarization-reconfigurable omnidirectional antenna combining dipole and loop radiators," IEEE Antennas Wireless Propag. Lett., vol. 12, pp. 1102-1105, Sept. 2013.

[14] W. Lin and R. Ziolkowski, "Compact, omni-directional, circularly-polarized mm-Wave antenna for device-to-device (D2D) communications in future 5G cellular systems," in Global Symp. on Millimeter-Waves, pp. 115-116, Hong Kong, China, May 2017.

[15] S. Ranvier et al., "Low-cost planar omnidirectional antenna for mm-wave applications," IEEE Antennas and Wireless Propag. Lett., vol. 7, pp. 521-523, Aug. 2008.

[16] Y. Liu, M.C.E. Yagoub, and M. Nassor, "Omni-directional antenna array with improved gain for 5G communication systems," in 2020 IEEE USNC-CNC-URSI North American Radio Science Meeting (Joint with AP-S Symp.), pp. 33-34, Montreal, QC, Canada, July 2020,

[17] K. Fan, Z. Hao, Q. Yuan, J. Hu, G. Q. Luo, and W. Hong, "Wideband horizontally polarized omnidirectional antenna with a conical beam for millimeter-wave applications," IEEE Trans. Antennas and Propag., vol. 66, pp. 4437-4448, Sept. 2018.

[18] S. Park, D. Shin, and S. Park, "Low side-lobe substrate-integrated-waveguide antenna array using broadband unequal feeding network for millimeter-wave handset device," IEEE Trans. Antennas and Propag., vol. 64, pp. 923-932, March 2016.

[19] A. Zhao and F. Ai, "5G mm-wave antenna array based on T-slot antenna for mobile terminals," in IEEE Asia-Pacific Conf. on Antennas and Propag., pp. 476-477, Auckland, New Zealand, Aug. 2018.

[20] S. Ranvier et al., "Low-cost planar omnidirectional antenna for mm-wave applications," IEEE Antennas Wireless Propag. Lett., vol. 7, pp. 521-523, Aug. 2008.

[21] Y. Liu, C. Deng, X. Lu, H. Sun and L. Shi, "Study of the improved mm-wave omni-directional microstrip antenna," in 2006 7th Int. Symp. on Antennas, Propag. \& EM Theory, Guilin, 2006, pp. 1-4. 